\title{
Phosphorylation via PKC regulates the function of the Drosophila odorant co-receptor
}

\author{
Vardanush Sargsyan, Merid Negash Getahun, Sofía Lavista Llanos, Shannon B. Olsson, Bill S. Hansson and \\ Dieter Wicher*
}

Evolutionary Neuroethology, Max Planck Institute for Chemical Ecology, Jena, Germany

Edited by:

Alexander Borst, Max Planck Institute of Neurobiology, Germany

Reviewed by:

Mala Shah, University of London, UK Shaoyu Ge, SUNY Stony Brook, USA Esther Alcorta, University of Oviedo, Spain

\section{${ }^{*}$ Correspondence:}

Dieter Wicher, Max Planck Institute

for Chemical Ecology,

Hans-Knöll-Street 8, D-07745 Jena,

Germany.

e-mail:dwicher@ice.mpg.de
Insect odorant receptors (ORs) have a unique design of heterodimers formed by an olfactory receptor protein and the ion channel Orco. Heterologously expressed insect ORs are activated via an ionotropic and a metabotropic pathway that leads to CAMP production and activates the Orco channel. The contribution of metabotropic signaling to the insect odor response remains to be elucidated. Disruption of the $\mathrm{G}_{q}$ protein signaling cascade reduces the odor response (Kain et al., 2008). We investigated this phenomenon in HEK293 cells expressing Drosophila Orco and found that phospholipase C (PLC) inhibition reduced the sensitivity of Orco to cAMP. A similar effect was seen upon inhibition of protein kinase $C$ (PKC), whereas PKC stimulation activated Orco even in the absence of cAMP. Mutation of the five PKC phosphorylation sites in Orco almost completely eliminated sensitivity to cAMP. To test the impact of PKC activity in vivo we combined single sensillum electrophysiological recordings with microinjection of agents affecting PLC and PKC function and observed an altered response of olfactory sensory neurons (OSNs) to odorant stimulation. Injection of the PLC inhibitor U73122 or the PKC inhibitor Gö6976 into sensilla reduced the OSN response to odor pulses. Conversely, injection of the PKC activators OAG, a diacylglycerol analog, or phorbol myristate acetate (PMA) enhanced the odor response. We conclude that metabotropic pathways affecting the phosphorylation state of Orco regulate OR function and thereby shape the OSN odor response.

Keywords: insect odorant receptor, Drosophila, Or83b, orco, G protein, cAMP, phosphorylation, single sensillum recording

\section{INTRODUCTION}

Olfaction in nematodes and vertebrates utilizes $\mathrm{G}$ protein-coupled receptor (GPCR) signaling. In insects, odorant receptor (OR) proteins share the seven-transmembrane topology of GPCRs but retain no sequence-similarity (Benton et al., 2006). Furthermore, they form heterodimers of a ligand-binding OR and an ubiquitous co-receptor such as Dmel/Orco (previously Or83b in Drosophila; Vosshall and Hansson, 2011), both of which are inversely oriented in the membrane compared to GPCRs (Benton et al., 2006; Lundin et al., 2007). On the other hand, G proteins are expressed in the dendrites of olfactory sensory neurons (OSNs) bearing the ORs (Boto et al., 2010), and Drosophila mutants with disturbed G protein signaling cascades show impaired odor processing (reviewed in Hansson et al., 2010). Here we concentrate on OR22a as ligandbinding receptor. This is probably the most well-investigated OR of D. melanogaster. It is expressed in large basiconic sensilla and is tuned to fruit volatiles (ethyl butyrate, ethyl hexanoate) emitted by, e.g., pineapple (Stensmyr et al., 2003; Hallem and Carlson, 2006).

Studies on insect OR function in heterologous expression systems provided evidence for a $\mathrm{G}$ protein-independent, ionotropic mode of action (Sato et al., 2008; Wicher et al., 2008). However, an additional metabotropic pathway has been shown to stimulate cAMP production, which in turn activates the ion channel-forming co-receptor protein Orco (Wicher et al., 2008). Manipulating the cAMP level changed the kinetics of the odor response in heterologous cells (Smart et al., 2008). Genetic manipulation of $G$ protein signaling in flies produced comparatively mild effects on odor responses (Yao and Carlson, 2010). However, the important role of stimulatory $\mathrm{G}$ proteins and subsequent cAMP signaling was recently demonstrated both in flies and in the heterologous expression system (Deng et al., 2011). A consensus model of these controversial results suggests a modulation of the ionotropic response by metabotropic pathways (Nakagawa and Vosshall, 2009).

In addition to cAMP signaling, $\mathrm{DAG} / \mathrm{IP}_{3}$ signaling may also play a role in insect olfaction (Krieger and Breer, 1999). Mutations in the Drosophila $d g q$ gene encoding the $\mathrm{G}_{\mathrm{q}} \alpha$ subunit produces flies with reduced responses to odor stimulation (Kain et al., 2008). The responses were further attenuated by additional mutations in plc21C, a gene encoding for a PLC $\beta$. In the present study, we investigate the effect of $\mathrm{G}_{\mathrm{q}}$ protein downstream signaling on heterologously expressed Orco proteins and demonstrate the relevance of these results using single sensillum electrophysiology in Drosophila OSNs combined with microinjection of compounds affecting the $\mathrm{G}_{\mathrm{q}}$ protein signaling cascade. 


\section{MATERIALS AND METHODS PKC MUTANT ORCO}

Or83b protein kinase C (PKC) phosphorylation mutants M1, M2, and Orco PKC synthetic genes were generated and subcloned into EcoRI/XhoI sites of pcDNA3.1(+) plasmid (eurofins MWG operon, Ebersberg, Germany) and directly used for cell transfections. Sequences were analyzed by doublestrand DNA sequencing (eurofins MWG operon) and point mutations for M1 (S159N, T250N, S289N), M2 (T327N, T371N), and Orco PKC (S159N, T250N, S289N, T327N, T371N) verified. Expression and membrane targeting of Orco PKC was demonstrated by immunofluorescence. HEK293 cells were cultured on glass coverslips, transfected, and fixed in 4\% PFA. Membranes were labeled with Texas Red-X conjugated wheat germ agglutinin (WGA; $5 \mu \mathrm{g} / \mathrm{ml}$, Invitrogen). Cells were subsequently incubated with a primary rabbit polyclonal antibody (1:1000) against Orco (kindly provided by Leslie Vosshall) and an Alexa488 (1:1000) secondary antibody. Confocal images were taken and analyzed by LSM 510 Meta (Carl Zeiss, Germany).

\section{CELL CULTURE AND TRANSFECTION Transient transfection}

HEK293 cells were cultured at a density of $\sim 2 \times 10^{4}$ per 35$\mathrm{mm}$ dish and transfected with $1 \mu \mathrm{g}$ Or83b-pcDNA3.1(-) or $1 \mu \mathrm{g}$ Or83b PKC-pcDNA3.1(+) using Roti-Fect transfection kit (Roth, Karlsruhe, Germany). In some experiments the partial Orco PKC mutants M1 and M2 were used (Figure 4A). To test for the role of $\mathrm{G}_{\mathrm{s}}$ and $\mathrm{G}_{\mathrm{q}}$ protein activation on Orco, cells were co-transfected with $1 \mu \mathrm{g} \mathrm{h} \beta_{2}$ adrenergic receptor $\left(\beta_{2} \mathrm{AR}\right) / \mathrm{pCMV6-XL5}$ (Origene, Rockville, MD, USA) and with $1 \mu \mathrm{g} \mathrm{h} \alpha_{1}$ adrenergic receptor $\left(\alpha_{1} \mathrm{AR}\right) / \mathrm{pCMV6}$-XL4 (Origene). In all preparations with transient transfection cells were co-transfected with $0.5 \mu \mathrm{g}$ EGFP; for electrophysiological experiments we only used cells showing GFP fluorescence (when illuminated at $470 \mathrm{~nm}$ ) as indicator of putative OR expression.

\section{Stable transfection}

The open reading frame of Orco was PCR-amplified using gene specific primers with restriction sites for XhoI and HindIII and cloned into the pcrII TA-cloning vector (Invitrogen, Carlsbad, CA, USA). The identity of the insert was sequenced in full length to verify identity and integrity and subcloned into the pcDNA3.1(+) expression vector via the integrated restriction sites. The resultant construct was verified by sequencing. Flp-In ${ }^{\mathrm{TM}}-\mathrm{T}-\operatorname{Rex}^{\mathrm{TM}} 293$ cells held in DMEM (high glucose, with 1-Glutamine)/Ham's F12 (with 1-Glutamine; PAA, Pasching, Austria) $+10 \%$ FCS were transfected with OR 83b pc DNA 3.1 using Fugene (Invitrogen) according to the manufacterer's protocol. 24-h post-transfection $800 \mathrm{mg} / \mathrm{l}$ of G418 were added to the medium. After 12 weeks, clones were separated and tested for activity using measurements of channel activity as described (Wicher et al., 2008). The clone exhibiting the highest sensitivity to 8-bromo-cAMP was maintained under antibiotic selection and used for this study.

\section{PATCH-CLAMP ELECTROPHYSIOLOGY}

Ion currents in HEK293 cells were measured at room temperature using whole-cell patch-clamp with appropriate compensation of series resistance and of capacitive currents. Additional experiments were performed in the inside-out configuration. Current measurements and data acquisition were performed using an EPC9 patch-clamp amplifier controlled by PatchMaster software (both HEKA Elektronik, Lambrecht, Germany). Patch-clamp pipettes were fabricated from borosilicate capillaries. Pipettes for whole-cell recordings had resistances of 2-4 M $\Omega$ for excised-patch recordings the pipette resistance was up to $15 \mathrm{M} \Omega$.

The pipette solution contained (in $\mathrm{mM}$ ) $140 \mathrm{KCl}, 4 \mathrm{NaCl}, 2.2$ $\mathrm{CaCl}_{2}$, 2 Mg-ATP, $0.05 \mathrm{Na}$-GTP, 5 EGTA, 10 HEPES (pH 7.3), and the bath solution contained (in $\mathrm{mM}$ ) $135 \mathrm{NaCl}, 5 \mathrm{KCl}, 1$ $\mathrm{CaCl}_{2}, 1 \mathrm{MgCl}_{2}, 10 \mathrm{HEPES}, 10$ glucose ( $\mathrm{pH} 7.4$ ). For recordings of inside-out patches the pipette solution was used for the bath and vice versa.

With the exception of GTP- $\gamma-S$, and GDP- $\beta-S$, which were applied via the patch pipette, all substances were applied to the bath using either a bath perfusion system (BPS4 from ALA, NY, USA) or a rapid solution changer (RSC160 from Biologic, Claix, France) which were controlled by the PatchMaster software (HEKA Elektronik).

For data analysis the software IgorPro (WaveMetrics, Lake Oswego, OR, USA) or Prism 4 (Graph Pad Software, San Diego, CA, USA) were used.

\section{EXTRACELLULAR SINGLE SENSILLUM RECORDING AND MICROINJECTION}

Recording and injection protocols were performed on Or22aGAL4; UAS-CD8-GFP Drosophila melanogaster flies expressing membrane tagged GFP in Or22a-OSNs. Two- to 5-day-old adults were fixed dorsally to a microscope slide. Compounds and concentrations for injection were diluted in receptor lymph solution (Kaissling and Thorson, 1980) as follows: U73122 (0.5 mM), Gö6976 (0.5 mM), OAG (0.1 mM), PMA (0.1 mM). Note that due to a dilution effect, concentrations of injected agents were $100 \times$ the concentration used in whole-cell preparations. A microinjection setup consisting of a dual-pump system was used to inject agents via air pressure through the microelectrode holder and into the sensillum lymph. For odor stimulation, $10 \mu \mathrm{l}$ of ethyl butyrate (99\%, Sigma, Munich, Germany) in hexane (10 ng/ $\mu$ l; 99\%, Fluka Analytical, Buchs, Switzerland) was pipetted onto $1 \mathrm{~cm}$ diameter filter paper disks and placed in disposable Pasteur pipettes. Odor stimuli were delivered at $0.5 \mathrm{l} / \mathrm{min}$ into a $1.0 \mathrm{l} / \mathrm{min}$ humidified air stream.

Sensilla were localized at $1000 \times$ magnification and an $\mathrm{Ag} / \mathrm{AgCl}$ coated silver wire inserted into a sharpened glass capillary used to detect the extracellular analog signals originating from the OSNs. Action potentials were extracted digitally according to top-top amplitudes using Syntech Auto Spike 32 software. Cell activities were recorded for approximately $20 \mathrm{~s}$ before an initial $0.5 \mathrm{~s}$ stimulation with ethyl butyrate. Microinjection commenced at $100 \mathrm{~s}$, and cells were again stimulated with an 0.5 -s odor pulse after approximately $300 \mathrm{~s}$. Responses of the larger amplitude Or22a-carrying cell were analyzed for $1500 \mathrm{~ms}$ after stimulus onset. For response kinetics, spike frequency ratios were analyzed as peri-stimulus time histograms (PSTHs) in $25 \mathrm{~ms}$ bins by dividing each $25 \mathrm{~ms}$ instantaneous spike frequency by the average pre-stimulus frequency over $2 \mathrm{~s}$ to give a normalized ratio for each time point. Areas under the PSTH curve were calculated for the stimulus $(500 \mathrm{~ms})$ 
and total response (1350 ms) windows respectively, adjusting for a $150 \mathrm{~ms}$ mechanical stimulus delay. These values were divided by time to establish a normalized frequency average for each response. Mann-Whitney $U$ tests compared treatments with the control (receptor lymph ringer) after injection. All analyses were performed using PASW (SPSS) v. 18 software.

\section{CHEMICALS}

8-Bromo-cAMP, 8-bromo-cGMP, dl-isoproterenol hydrochloride (ISO), dl-Norepinephrine hydrochloride (NE), ethyl butyrate (Etb), forskolin, GTP- $\gamma$-S, GDP- $\beta$-S, phorbol 12-myristate 13acetate (PMA), and 9-(tetrahydro-2-furanyl)-9H-purin-6-amine (SQ22536) were obtained from Sigma (Taufkirchen, Germany); U73122, U73343 and Gö6976 from Calbiochem (Darmstadt, Germany); 1-oleoyl-2-acetyl-sn-glycerol (OAG) from Alexis (Lörrach, Germany).

\section{RESULTS}

Mutant flies with disrupted $\mathrm{G}_{\mathrm{q}}$ protein/phospholipase C (PLC) signaling cascade show reduced odor responses (Kain et al., 2008). When seeking the molecular mechanism by which PLC inhibition affects the odor response, the most parsimonious assumption is that PLC targets the OR complex itself. As multiple receptors are affected by $\mathrm{G}_{\mathrm{q}}$ protein disruption (Kain et al., 2008), the ubiquitous Orco would be a good target candidate. In human embryonic kidney (HEK293) cells co-expressing Or22a and Orco, we have previously observed that intracellular application of the non-hydrolysable G protein inhibitor GDP- $\beta$-S reduces the sensitivity of the receptor dimers to ethyl butyrate, a key ligand (Wicher et al., 2008). G protein inhibition prevented odor-induced cAMP production and consequent activation of Orco via the metabotropic pathway. Here, we asked whether inhibition of $G$ proteins could affect the sensitivity of Orco to cAMP. Using the voltage-clamp technique in the whole-cell configuration, we perfused HEK293 cells expressing Orco with the non-hydrolysable GDP analog GDP- $\beta-S$ via a patch pipette. Stimulation of Orco by bath application of the membrane-permeable cAMP analog 8-bromo-cAMP could - even at the highest concentrations induce only a weak membrane current (Figure 1B). Without GDP- $\beta$-S in the pipette, 8 -bromo-cAMP induced a current in a concentration-dependent manner (Figure 1B). Unexpectedly, permanent stimulation of $\mathrm{G}$ proteins with the non-hydrolysable GTP analog GTP- $\gamma$-S induced a current even in the absence of 8-bromo-cAMP (Figures 1A,B). Subsequent application of 8bromo-cAMP further enhanced this current. However, there was less current production by 8-bromo-cAMP than under control conditions (287 pA vs. 550 pA; Figure 1B), indicating that the pool of channels available for activation by 8 -bromo-cAMP is reduced due to pre-activation by GTP- $\gamma-S$.

To assess whether $G$ proteins have a direct effect on Orco and whether there is any subtype-specificity, we co-expressed Orco together with the $\beta_{2}$-adrenergic receptor $\left(\beta_{2}-\mathrm{AR}\right)$, which activates $\mathrm{G}_{\mathrm{s}}$ proteins, and with the $\alpha_{1}$-adrenergic receptor $\left(\alpha_{1}-\mathrm{AR}\right)$, which activates $\mathrm{G}_{\mathrm{q}}$ proteins. Stimulation of $\beta_{2}$-AR with $10 \mu \mathrm{M}$ isoproterenol induced currents of comparable size to those obtained after application of $1 \mu \mathrm{M} 8$-bromo-cAMP (relative current: $1.2 \pm 0.2$; $n=5$ ). Preincubation of cells with the adenylyl cyclase inhibitor

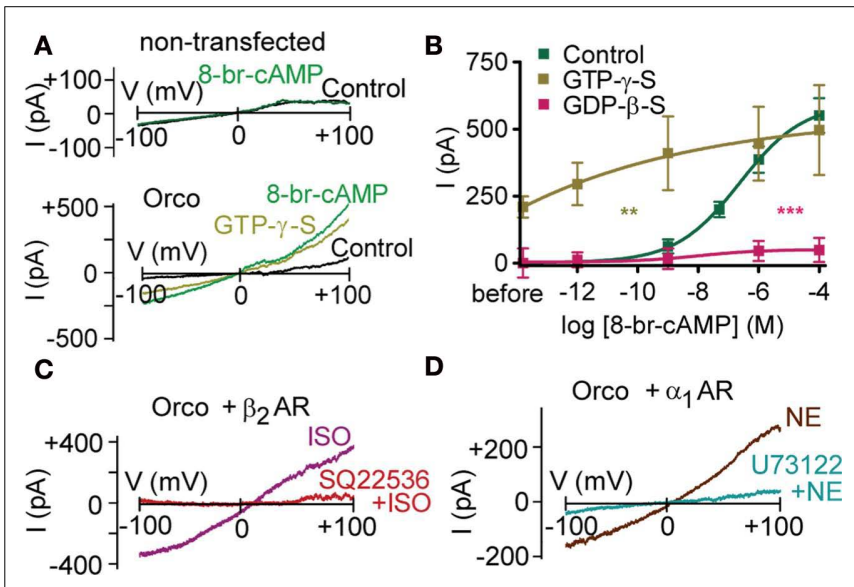

FIGURE 1 | G protein activity regulates the cAMP effect on Orco. (A) Whole-cell current response in a non-transfected HEK293 cell on a voltage ramp from -100 to $+100 \mathrm{mV}$ after breaking into the cell (Control) and $2 \mathrm{~min}$ after application of $100 \mu \mathrm{M}$ 8-br-cAMP (top), and a HEK293 cell expressing Orco after breaking into the cell (Control), after perfusion with GTP- $\gamma$-S $(500 \mu \mathrm{M})$ to activate $\mathrm{G}$ proteins, and $2 \mathrm{~min}$ after application of $100 \mu \mathrm{M}$ 8-br-cAMP (bottom). Note that 8-br-cAMP fails to produce a current in non-transfected cells. (B) Concentration-response curve for 8-br-cAMP-induced Orco currents, measured at $-100 \mathrm{mV}$ in the whole-cell mode with standard pipette solution (Control) and a solution containing GTP- $\gamma-S(500 \mu \mathrm{M})$ to activate or GDP- $\beta-S(500 \mu \mathrm{M})$ to inactivate G proteins, respectively. The current "before" is the difference between GTP- $\gamma$-S and Con described in (A). (Control, $n=11$; GTP- $\gamma-\mathrm{S}, n=8,{ }^{*} P$ to

Control $<0.01$; GDP- $\beta-S, n=9,{ }^{*}{ }^{*} P<0.001$ ). (C) Test for the role of $\mathrm{G}_{\mathrm{s}}$ protein activation on Orco. Current responses in a cell expressing Orco and the $\beta_{2}$ adrenergic receptor $\left(\beta_{2}\right.$ AR) to isoproterenol stimulation $(10 \mu \mathrm{M})$ in the absence (ISO) and $6 \mathrm{~min}$ after application of SO22536 (200 $\mu \mathrm{M})$. Curves represent differences (2 min ISO - before ISO). (D) Test for the role of $\mathrm{G}_{\mathrm{q}}$ protein activation on Orco. Currents evoked in a cell expressing Orco and the $\alpha_{1}$ adrenergic receptor $\left(\alpha_{1} A R\right)$ by norepinephrine (NE) stimulation $(1 \mu \mathrm{M})$ in the absence and $8 \mathrm{~min}$ after application of the PLC inhibitor U73122 (10 $\mu \mathrm{M})$. Curves represent differences ( $2 \mathrm{~min} \mathrm{NE}-$ before NE).

SQ22536 $(200 \mu \mathrm{M})$ prevented current production by isoproterenol (relative current: $-0.1 \pm 0.1 ; n=5$; Figure 1C). Thus, neither the $G_{s} \alpha$ subunit nor the $\beta \gamma$ subunit complex was able to activate Orco in the absence of cAMP.

Stimulation of $\alpha_{1}$-AR with $1 \mu \mathrm{M}$ norepinephrine induced currents of $1.6 \pm 0.3(n=5)$, normalized to the $1 \mu \mathrm{M}$ 8-bromo-cAMP response. To test for a direct $\mathrm{G}$ protein effect, PLC was inhibited with $\mathrm{U} 73122(10 \mu \mathrm{M})$. Under these conditions, norepinephrine failed to elicit a current (relative current: $-0.2 \pm 0.2 ; n=7$; Figure 1D), indicating that neither the $\mathrm{G}_{\mathrm{q}} \alpha$ subunit nor the $\beta \gamma$ subunit complex could activate Orco alone. The activation of coexpressed Orco upon stimulation of $\alpha_{1}$-AR is remarkable as it for the first time demonstrates that Orco can be metabotropically activated independent of cyclic nucleotides. There are thus at least two independent signaling pathways capable of producing Orco currents.

To examine whether inhibition of PLC activity could account for the reduced cAMP-sensitivity of Orco with GDP- $\beta-S$, we tested the effect of 8 -bromo-cAMP in the presence of U73122. We then observed only marginal responses, even at the highest concentration of 8-bromo-cAMP (Figures 2A,C). U73343, an 
inactive analog of U73122, did not suppress the 8-bromo-cAMP effect $(P=0.91 ; n=8)$. The $\mathrm{G}$ protein activity-dependence of the Orco response to cAMP is therefore related to either PLC activity or a downstream process, i.e., the response of Orco to cAMP requires some basal PLC activity. To maintain catalytic activity of PLC, physiologically free $\mathrm{Ca}^{2+}$ levels are necessary (Rebecchi and Pentyala, 2000). We thus tried to stimulate Orco using a $\mathrm{Ca}^{2+}$-free pipette solution, and we recorded only marginal responses to 8-bromo-cAMP $(5 \mu \mathrm{M} ; 31 \pm 17 \mathrm{pA} ; n=9)$ or forskolin $(10 \mu \mathrm{M} ; 95 \pm 41 \mathrm{pA} ; n=8)$ compared with those obtained with standard pipette solution (cAMP: $398 \pm 67 \mathrm{pA}$; $n=11$; forskolin: $697 \pm 67 \mathrm{pA} ; n=9$ ).

Inhibition of PLC activity prevents $\mathrm{PIP}_{2}$ cleavage and subsequent $\mathrm{IP}_{3}$ and DAG production. We asked which of these effects could account for the observed depression of current production by cAMP. Mimicking PIP $_{2}$ accumulation due to PLC inhibition using bath application of $\mathrm{PIP}_{2}$ with inside-out patches did not significantly reduce the cAMP effect $(5 \mu \mathrm{M}$ cAMP enhanced the background current of $3.6 \pm 0.7 \mathrm{pA}$ by $10 \pm 2.7 \mathrm{pA} ; n=8$ ). Thus, the $\mathrm{PIP}_{2}$ cleavage products may instead be critical for the cAMPsensitivity of Orco. Even though $\mathrm{IP}_{3}$ activated a tiny current $(\sim 0.5 \mathrm{pA}$ at $5 \mu \mathrm{M})$, this current was too weak to rescue the

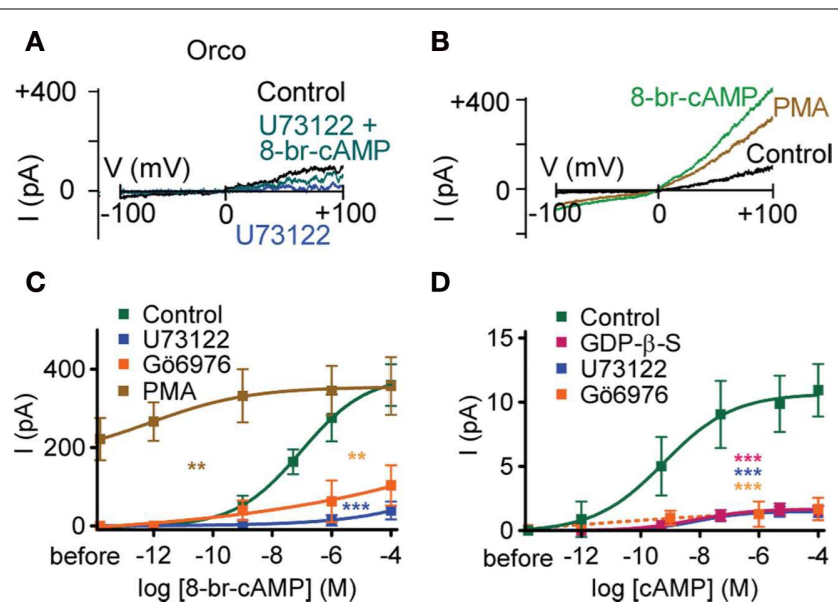

FIGURE 2 | Phospholipase $\mathbf{C}$ and protein kinase $\mathbf{C}$ activity regulates the cAMP effect on Orco. (A) Current responses in a HEK293 cell expressing Orco on a voltage ramp from -100 to $+100 \mathrm{mV}$ after breaking into the cell (Control), after application of the PLC inhibitor U73122 (10 $\mu \mathrm{M})$, and after application of 8-br-cAMP $(100 \mu \mathrm{M})$ in presence of U73122. (B) Current responses in a cell expressing Orco before (Control) and after PMA (1 $\mu \mathrm{M})$ and 8-br-cAMP stimulation (100 $\mu \mathrm{M})$. (C) Concentration-response for 8-br-cAMP-induced Orco currents, measured as described in (B) with a standard bath solution (Control) and a solution containing U73122 (10 $\mu \mathrm{M})$, the PKC inhibitor Gö6976 $(2 \mu \mathrm{M})$ or the PKC activator PMA (1 $\mu \mathrm{M})$, respectively. (Control, $n=10$; U73122, $n=7$, *** $P<0.001$; Gö6976, $n=11$, ${ }^{*} P<0.01$; PMA $\left., n=11,{ }^{*} P<0.01\right)$. (D) Concentration-response curves for cAMP-induced currents in inside-out patches from cells expressing Orco. Data represent maximum mean currents at $-60 \mathrm{mV}$ produced under control conditions and with $500 \mu \mathrm{M}$ GDP- $\beta-S, 10 \mu \mathrm{M}$ U73122 or $1 \mu \mathrm{M}$ Gö6976 in the bath. (Control, $n=13 ;$ GDP- $\beta-S, n=17,{ }^{* *} P<0.001$; U73122, $n=16,{ }^{* *} P<0.001$; Gö6976, $\left.n=10, * * * P<0.001\right)$. The continuous curves are Hill fits described by $\mathrm{EC}_{50}$ values of $677 \mathrm{pM}, 33 \mathrm{nM}$ and $10 \mathrm{nM}$ and Hill coefficients of $0.40,0.33$, and 0.51 for the control, GDP- $\beta-S$ and U73122, respectively.
U73122-inhibited current, and it likely reflects the activation of an endogeneous HEK293 cell channel (Bugaj et al., 2005). By contrast, the DAG analog OAG enhanced the membrane current upon PLC inhibition (at $100 \mu \mathrm{M}$ from $0.8 \pm 0.2 \mathrm{pA}$ to $2.6 \pm 0.7 \mathrm{pA}$; $n=6$ ), and partially restored sensitivity to cAMP (at $100 \mu \mathrm{M}$ to $5.4 \pm 1 \mathrm{pA} ; n=6)$. DAG levels thus appear to control the response of Orco to cAMP. As DAG activates PKC, we assessed whether inhibition of PKC would mimic the effect of PLC inhibition. Application of 8-bromo-cAMP after preincubation of cells with the PKC inhibitor Gö6976 produced only weak whole-cell current responses that did not differ significantly from those obtained with U73122 (Figure 2C). On the other hand, activation of PKC with phorbol myristate acetate (PMA) induced an Orco current, while subsequent application of 8-bromo-cAMP caused only a mild further current increase (Figures 2B,C).

The concentration-response curve for whole-cell current activation by 8 -bromo-cAMP indicates that Orco is highly sensitive to cAMP at physiological $\mathrm{Ca}^{2+}$ levels (Figures 2B,C). To demonstrate this directly we tested the effect of cAMP on inside-out patches. Intriguingly, current activation even after fast application of cAMP develops slowly and with a delay (Figure 3). The activation process thus differs from classical gating such as in $\mathrm{CNG}$ channels. The concentration-response curve obtained was characterized by $\mathrm{EC}_{50}=0.7 \mathrm{nM}$ cAMP and a Hill coefficient $=0.40$ (Figure 2D). Therefore, Orco is at least four orders of magnitude more sensitive to cAMP than the most cAMP-sensitive CNG channel (Dhallan et al., 1990). As in the whole-cell experiments, GDP- $\beta$-S, U73122, and Gö6976 drastically reduced the responses to cAMP (Figure 2D).

These results suggest that PKC activity plays a central role in controlling Orco function, and especially the sensitivity to cAMP. The Orco protein bears five PKC phosphorylation sites (Figure 4A), three in the intracellular loop 2 (IC2), T250, S289 and T327, and two with putative extracellular localization (S159 in EC2; T371 in EC3). To confirm the role of PKCmediated phosphorylation, we produced an Orco PKC mutant with serine/threonine to asparagine replacements at all PKC sites (Figure 4A). This mutant was expected to mimic the Orco wild type in the presence of PLC or PKC inhibitors for both background activity and stimulation by cAMP. Odorant receptor heterodimers such as Or22a/Orco show some background activity even in the absence of odor stimuli (Wicher et al., 2008). Inside-out patches from HEK293 cells expressing Orco conducted a significantly higher resting current than those from non-transfected cells

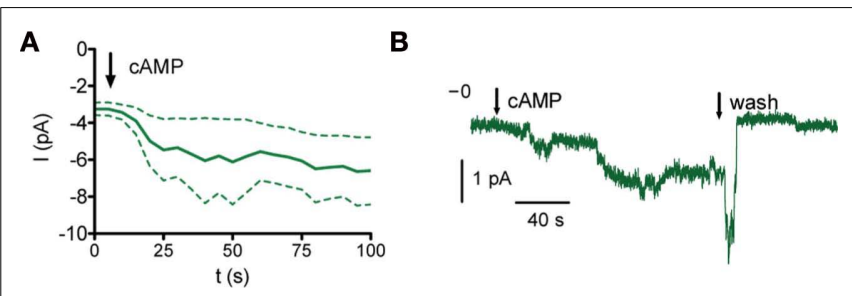

FIGURE 3 | Time course of Orco current activation. (A) Current response upon cAMP stimulation (bold, mean of four excised patches, inside-out configuration, dashed, SEM). (B) Sample trace of an inside-out patch. Arrows mark application or wash of 500 pM cAMP as indicated. 


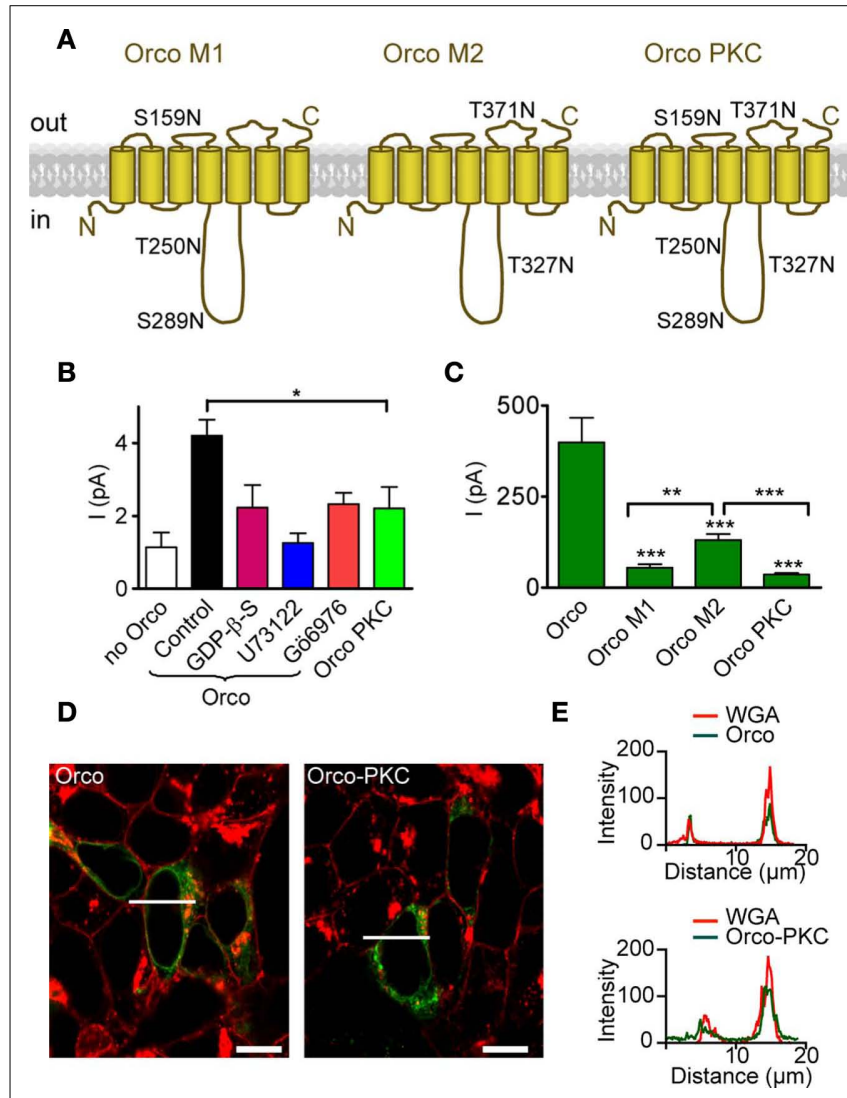

FIGURE 4 | Mutation of PKC phosphorylation sites in Orco reduces the resting current and CAMP responses. (A) Scheme of Orco topology with mutations of PKC sites indicated in the three mutants Orco M1, Orco M2, and Orco PKC. Two sites are predicted to be in extracellular loops (ECL2 and ECL3) while three sites are in the intracellular loop 2. (B) Mean inward currents in inside-out patches from non-transfected HEK293 cells $(n=12)$, cells expressing Orco and Orco PKC, measured at $-60 \mathrm{mV}$ without stimulation. The constitutive current through Orco (Control, $n=43$ ) was reduced by GDP- $\beta$-S; $\left(500 \mu \mathrm{M}, n=13,{ }^{*} P<0.05\right.$, Student's $t$-test.), U73122 $\left(10 \mu \mathrm{M} ; n=10,{ }^{*} P<0.01\right)$, and Gö6976 $\left(1 \mu \mathrm{M} ; n=8,{ }^{*} P<0.05\right)$, and it is significantly larger than in patches from non-transfected HEK293 cells (***P<0.001). Compared with Orco PKC containing patches $(n=15)$, only the Orco current in the control is significantly larger $\left({ }^{*} P=0.02\right)$. (C) Whole-cell current responses to $5 \mu \mathrm{M}$ 8-br-cAMP in cells expressing Orco (WT) or the three PKC mutants, measured at $-100 \mathrm{mV}$. (WT, $n=11$; M1, $n=10 ; \mathrm{M} 2, n=15$; Orco PKC, $n=11 ;-4,{ }^{* * * P}<0.001$; Student's $t$-test). Currents in $\mathrm{M} 2$ expressing cells are significantly larger than in $\mathrm{M} 1$ $\left({ }^{* *} P=0.002\right)$ or Orco PKC (***P<0.001) expressing cells. Error bars represent SEM. (D) Confocal micrographs of HEK293 cells transfected with Orco and Orco PKC. Green, immunofluorescence; red, Texas-red fluorescence of wheat germ agglutinine (WGA) labeled plasma membrane; bar, $10 \mu \mathrm{m}$; line indicates position of intensity profile shown in (E). (E) Intensity profile of Orco and Orco PKC immunfluorescence and membrane staining (WGA-Texas-red) in two cells displayed in (D). Colocalization of fluorescence signals indicates membrane insertion of the Orco proteins.

(Figure 4B), indicating that Orco is spontaneously active even in the absence of cAMP. The resting current was reduced by GDP- $\beta-S$, U73122, and Gö6976 (Figure 4B). For the PKC phosphorylation mutant Orco PKC, the resting current was similar to nontransfected cells and Orco-expressing cells in the presence of these inhibitors, and was significantly lower than for Orco-expressing cells under control conditions (Figure 4B). Compared to native Orco stimulation, activation of the mutant with 8-bromo-cAMP in the whole-cell configuration or cAMP in inside-out patches produced very weak responses (Figures 4C, 5 and 6). Similarly, PMA failed to elicit a current in the absence of 8-bromo-cAMP (Figure 5C).

To confirm that impaired membrane targeting of Orco PKC did not account for the small current production by cAMP, we tested the distribution of Orco and Orco PKC immunofluorescence in the HEK293 cells (Figure 4D). Comparison of immunostaining with plasma membrane staining indicated that both Orco and Orco PKC were localized within the membrane (Figures 4D,E).

We also designed two partial mutants (Figure 4A), mutant 1 (S159N, T250N, S289N) and mutant 2 (T327N, T371N) which both showed no resting activity. We next asked how their response to 8-bromo-cAMP compared to that of Orco and Orco PKC (Figure 4C). The weakest response was seen in the complete mutant Orco PKC; the response obtained with mutant 2 was significantly stronger than that of mutant 1 and Orco PKC itself (Figure 4C). This indicates that the various

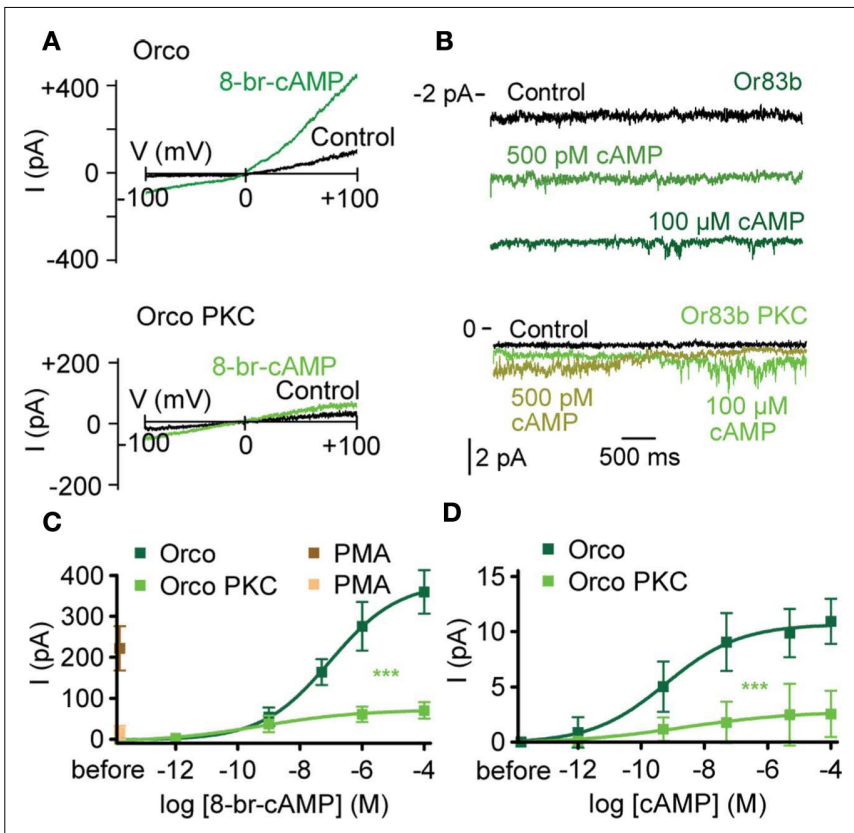

FIGURE 5 | Complete mutation of PKC phosphorylation sites in Orco disrupts cAMP responses. (A) Whole-cell current response in a cell expressing Orco (top) and Orco PKC (bottom) on a voltage ramp from -100 to $+100 \mathrm{mV}$ after breaking into the cell (Con) and $2 \mathrm{~min}$ after application of 8-br-cAMP $(100 \mu \mathrm{M})$. (B) Current recordings from excised patches (inside-out configuration) of a cell expressing Orco (top) and Orco PKC (bottom) after breaking into the cell (Control) and $2 \mathrm{~min}$ after application of cAMP at $500 \mathrm{pM}$ and $100 \mu \mathrm{M}$, respectively. Patches were held at $-60 \mathrm{mV}$, bars indicate current level. (C) Concentration-response curve for 8-br-cAMP-induced Orco and Orco PKC whole-cell currents, measured at $-100 \mathrm{mV}$. (Orco, $n=10$; Orco PKC, $\left.n=13,{ }^{* *} P<0.001\right)$. PMA $(1 \mu \mathrm{M})$ was applied in the absence of 8-br-cAMP. (Orco, $n=11$; Orco PKC, $n=10$ ). (D) Concentration-response curve for CAMP-induced currents in inside-out patches from cells expressing Orco and Orco PKC. Data represent maximum mean currents, measured at $-60 \mathrm{mV}$. (Orco, $n=13$; Orco PKC, $\left.n=13,{ }^{*} * * 0.001\right)$ 


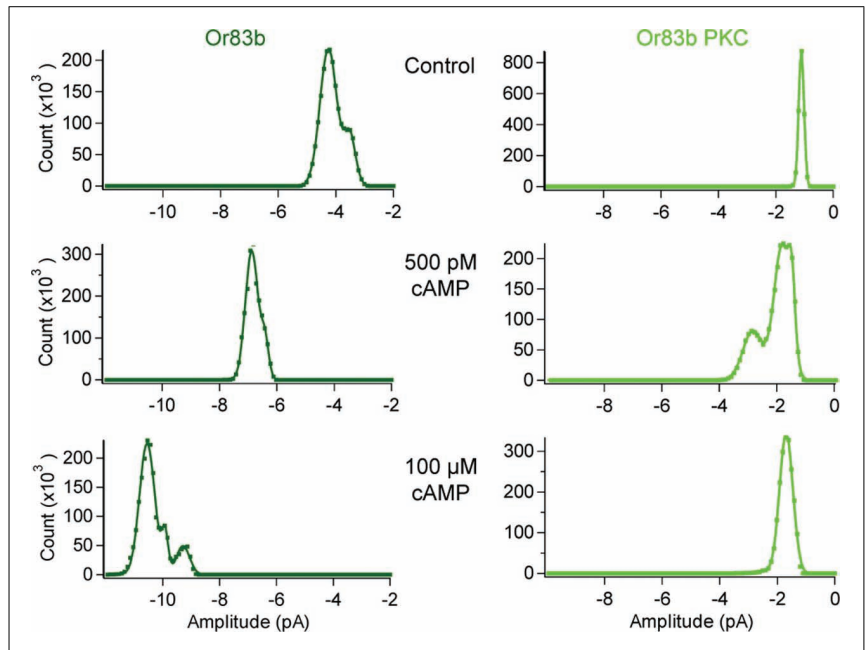

FIGURE 6 | cAMP responses in Orco and Orco PKC. All-point histograms for the data shown in Figure 5B. Currents from inside-out patches from a cell expressing Orco (top) and Orco PKC (bottom) after breaking into the cell (Control) and 2 min after application of CAMP at $500 \mathrm{pM}$ and $100 \mu \mathrm{M}$, respectively. Patches were held at $-60 \mathrm{mV}$, currents were recorded for $5 \mathrm{~s}$ (20 repetitions).
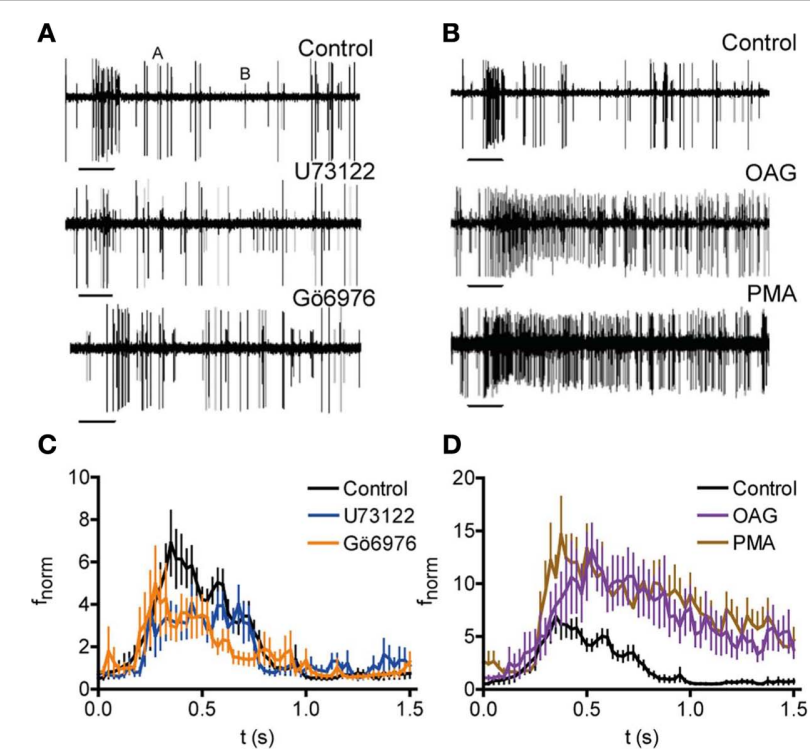

FIGURE 7 | Modulation of PKC-mediated phosphorylation affects the odor response of Drosophila OSNs. (A,B) Recordings of neuronal activity before and after Etb stimulation ( $-5 \mathrm{v} / \mathrm{v} ; 0.5 \mathrm{~s}$, bars) following injection of indicated compounds (Control, injection of receptor lymph solution). Etb stimulation enhances the activity of the $A$ neuron but not of the $B$ neuron. Inhibition of PLC (U73122) and PKC (Gö6976) reduces the Etb response of the $A$ neuron (A) whereas activation of PKC (OAG, PMA) enhances the Etb response (B). (C) Normalized spike frequency $\left(f_{\text {norm }}\right)$ of the A neuron upon Etb stimulation $(0-0.5 \mathrm{~s}$, at $-5 \mathrm{v} / \mathrm{v})$ after injection of receptor lymph solution (Control; $n=11)$, U73122 ( $n=13)$, or Gö6976 ( $n=12)$. Both treatments reduce the odor response (for $P$ see text). (D) $f_{\text {norm }}$ as described in (C) after injection of ringer solution (Control; $n=11)$ OAG $(n=8)$ or PMA $(n=10)$.

phosphorylation sites contribute to cAMP-sensitivity in additive manner, and the extent of Orco response to cAMP stimulation may thus be regulated by the degree of its phosphorylation via PKC.

To test whether PKC phosphorylation affects the odor response of OSNs in the fly, we combined extracellular recording of OSN activity upon odor stimulation with injection of compounds affecting PLC/PKC activity. For these experiments, a microelectrode was inserted into the antenna near the base of large basiconic ab3 sensilla housing OSNs expressing the receptor protein Or22a. These neurons were localized under fluorescence using the GAL4UAS system to drive GFP expression in Or22a expressing neurons (Dobritsa et al., 2003). Stimulation with ethyl butyrate (Etb; $\log [-5]$ dilution) accelerated the firing frequency of the Or22a expressing neuron ( $\mathrm{A}$ in Figures $7 \mathbf{A}, \mathbf{B} ; f_{\text {norm }}=3.72 \pm 0.73$, stimulus window, $f_{\text {norm }}=2.37 \pm 0.22$, total response, $n=11$ ) without affecting the other neuron B), as shown by Hallem et al. (2004). Injection of the PLC inhibitor U73122 into sensilla diminished the response to Etb in comparison to sham injection (Figures 7A,C; $f_{\text {norm }}=2.52 \pm 0.43$, stimulus window, $n=13 ; P=0.014$, MannWhitney $U$ ). By contrast, the inactive analog U73343 had no effect $\left(f_{\text {norm }}=4.67 \pm 1.04\right.$, stimulus window, $n=11, P=0.870$, Mann-Whitney $U$ ). The PKC inhibitor Gö6976 reduced the odor response in a manner similar to U73122 (Figures 7A,C; $f_{\text {norm }}=2.57 \pm 0.43$, stimulus window, $n=12$; $P=0.023$, MannWhitney $U$ ). By contrast, injection of the PKC activators OAG or PMA caused a robust increase of the Etb response (Figures 7B,D; $f_{\text {norm }}=7.06 \pm 1.45$ and $7.91 \pm 1.17$, respectively, total response, $n=8$ and $10 ; P=0.003$ and $<0.001$, Mann-Whitney $U)$.

\section{DISCUSSION}

Here we present evidence that the function of the odorant coreceptor Orco is controlled by its phosphorylation state via PKC. Regulation of ligand sensitivity by PKC phosphorylation has earlier been observed in, e.g., CNG channels. PKC activity can either enhance cGMP sensitivity (Müller et al., 1998) or reduce it (Müller et al., 2001). In the latter case the phosphorylation site was localized within the cGMP binding domain. Under normal physiological conditions the basal activity of PLC and PKC in HEK293 cells is sufficient to maintain Orco sensitivity to CAMP stimulation. Conditions leading to inhibition of these enzymes such as low free $\mathrm{Ca}^{2+}$ concentration suppress the activation of Orco by cAMP and thus may affect the odorant response. By contrast, high PLC/PKC activity would activate Orco independently of cAMP.

Inhibition of PLC function by GDP- $\beta$-S in HEK293 cells expressing Orco indicates constitutive activity of Orco- $\mathrm{G}_{\mathrm{q}}$ protein pairs. Constitutive activity of GPCR-G protein pairs was, for example, reported in other receptors such as thromboxane receptors (Chillar et al., 2010) and mutations in adrenergic receptors (Cotecchia, 2010). In insect ORs, constitutive activity causing a receptor current in the absence of stimuli occurs in various heterologously expressed receptor heterodimers (Sato et al., 2008; Wicher et al., 2008), as well as in solely expressed Orco (Figure 4B). For Orco, background activity of PLC or PKC seems to be sufficient to maintain a phosphorylation state required for a constitutive activity (Figure 4B).

Odor stimulation of Or22a, either solely expressed or coexpressed with Orco in HEK293 cells, activated $\mathrm{G}_{\mathrm{s}}$ proteins but not $\mathrm{G}_{\mathrm{q}}$ proteins (Wicher et al., 2008). If this is a general rule 
(which remains to be shown), odorant responses would not induce $\mathrm{G}_{\mathrm{q}}$ downstream signaling. This signaling cascade would thus be available to modulate OR function, for example by neuromodulators. The experiments using combined single sensillum recordings and microinjection demonstrate the significance of up- and down-regulation of PLC/PKC activity for the odor response of OSNs. Enzyme inhibition reduced the frequency dynamics of the OSN response, while stimulation of PKC produced a more robust and prolonged OSN response (Figure 7). A recent study utilizing similar extracellular recordings in transgenic flies with various $G$ protein mutations failed to see any effect of $G$ proteins on the in vivo olfactory response (Yao and Carlson, 2010). However, genetically manipulated animals could contain some counter-regulation of the metabotropic effects such as adaptation or up-regulation of regulating enzymes (e.g., phosphodiesterases). This can even occur during transient expression of constitutively active $\mathrm{G}$ proteins.

An unexpected result of the experiments with excised patches from HEK293 cells expressing Orco was the slow activation kinetics of the current after fast cAMP stimulation (Figure 3 ). The heterologous system may lack components that in vivo accelerate its activation. However, in the case that this slow time course of Orco activation would be similar in the OSNs, metabotropic signaling would not be able to contribute to the fast odor response of these neurons. For example, a 0.5 -s odor pulse gives rise to a response terminating after $2 \mathrm{~s}$ (Figure 7), whereas the response of Orco to cAMP took tens of seconds to develop (Figure 3).

\section{REFERENCES}

Benton, R., Sachse, S., Michnick, S. W., and Vosshall, L. B. (2006). Atypical membrane topology and heteromeric function of Drosophila odorant receptors in vivo. PLoS Biol. 4, e20. doi: 10.1371/journal.pbio.0040020

Boto, T., Gomez-Diaz, C., and Alcorta, E. (2010). Expression analysis of the $3 \mathrm{G}$-protein subunits, $\mathrm{G} \alpha, \mathrm{G} \beta$, and $\mathrm{G} \gamma$, in the olfactory receptor organs of adult Drosophila melanogaster. Chem. Senses 35, 183-193.

Bugaj, V., Alexeenko, V., Zubov, A., Glushankova, L., Nikolaev, A., Wang, Z., Kaznacheyeva, E., Bezprozvanny, I., and Mozhayeva, G. N. (2005). Functional properties of endogenous receptor- and store-operated calcium influx channels in HEK293 cells. J. Biol. Chem. 280, 16790-16797.

Chillar, A., Wu, J., Cervantes, V., and Ruan, K. H. (2010). Structural and functional analysis of the C-terminus of Gaq in complex with the human thromboxane A2 receptor provides evidence of constitutive activity. Biochemistry 49, 6365-6374.

Cotecchia, S. (2010). Constitutive activity and inverse agonism at the $\alpha$ (1a) and $\alpha(1 \mathrm{~b})$ adrenergic receptor subtypes. Meth. Enzymol. 485, 123-138.

Deng, Y., Zhang, W., Farhat, K. Oberland, S., Gisselmann, G., and Neuhaus, E. M. (2011). The stimulatory Galpha(s) protein is involved in olfactory signal transduction in Drosophila. PLoS ONE 6, e18605. doi: 10.1371/journal.pone.0018605

Dhallan, R. S., Yau, K. W., Schrader, K. A., and Reed, R. R. (1990). Primary structure and functional expression of a cyclic nucleotideactivated channel from olfactory neurons. Nature 347, 184-181.

Dobritsa, A. A., Van Der Goes Van Naters, W., Warr, C. G., Steinbrecht, R. A., and Carlson, J. R. (2003). Integrating the molecular and cellular basis of odor coding in the Drosophila antenna. Neuron 37, 827-841.

Hallem, E. A., and Carlson, J. R. (2006). Coding of odors by a receptor repertoire. Cell 125, 143-160.

Hallem, E. A., Ho, M. G., and Carlson, J. R. (2004). The molecular basis of odor coding in the Drosophila antenna. Cell 117, 965-979.

Hansson, B. S., Knaden, M., Sachse, S., Stensmyr, M. C., and Wicher, D. (2010). Towards plant-odorrelated olfactory neuroethology in Drosophila. Chemoecology 20, 51-61.

It must be noted that the compound microinjection mimicked metabotropic signaling processes initiated in a temporal domain before the odor stimulation.

Our study was not designed to determine whether insect ORs are mixed ionotropic and metabotropic receptors (Wicher, 2010) or metabotropically modulated ionotropic receptors (Nakagawa and Vosshall, 2009). However, the PKC mutant of Orco provides a useful tool to address this question in future investigations that assess the relationship between ionotropic and metabotropic signaling.

\section{CONCLUSION}

We have demonstrated a significant impact of the metabotropic pathway on olfactory response both in heterologous in vitro studies as well as in the Drosophila fly itself. Orco phosphorylation via PKC regulates OR sensitivity to cAMP, and therefore to odorants, and it may activate the receptor even in the absence of cAMP. This provides a powerful mechanism to adapt OR sensitivity not only via $\mathrm{G}_{\mathrm{q}}$ proteins but also via free $\left[\mathrm{Ca}^{2+}\right]_{\mathrm{i}}$ levels.

\section{ACKNOWLEDGMENTS}

This study was supported by the Max Planck Society (Vardanush Sargsyan, Sofía Lavista Llanos, Bill S. Hansson, Dieter Wicher), the International Max Planck Research School (Merid Getahun), and iChem (6th Programme of the EU; Shannon Olsson). We thank S. Bucks, S. Kaltofen, and R. Schäfer for technical assistance, E. Grosse-Wilde and M. C. Stensmyr for discussion.

Kain, P., Chakraborty, T. S., Sundaram, S., Siddiqi, O., Rodrigues, V., and Hasan, G. (2008). Reduced odor responses from antennal neurons of $\mathrm{Gq} \alpha$, phospholipase $\mathrm{C} \beta$, and rdgA mutants in Drosophila support a role for a phospholipid intermediate in insect olfactory transduction. J. Neurosci. 28, 4745-4755.

Kaissling, K.-E., and Thorson, J. (1980) "Insect olfactory sensilla: structural, chemical and electrical aspects of the functional organization," in Receptors for Neurotransmitters, Hormones and Pheromones in Insects, eds D. B. Sattelle, L. M. Hall, and J. G. Hildebrand (Amsterdam: Elsevier/NorthHolland), 261-282.

Krieger, J., and Breer, H. (1999). Olfactory reception in invertebrates. Science 286, 720-723.

Lundin, C., Kall, L., Kreher, S. A. Kapp, K., Sonnhammer, E. L., Carlson, J. R., Heijne, G., and Nilsson, I. (2007). Membrane topology of the Drosophila OR83b odorant receptor. FEBS Lett. 581, 5601-5604.

Müller, F., Bönigk, W., Sesti, F., and Frings, S. (1998). Phosphorylation of mammalian olfactory cyclic nucleotide-gated channels increases ligand sensitivity. J. Neurosci. 18, 164-173.
Müller, F., Vantler, M., Weitz, D., Eismann, E., Zoche, M., Koch, K. W., and Kaupp, U. B. (2001). Ligand sensitivity of the 2 subunit from the bovine cone cGMP-gated channel is modulated by protein kinase $\mathrm{C}$ but not by calmodulin. J. Physiol. 532, 399-409.

Nakagawa, T., and Vosshall, L. B. (2009). Controversy and consensus: noncanonical signaling mechanisms in the insect olfactory system. Curr. Opin. Neurobiol.19, 284-292.

Rebecchi, M. J., and Pentyala, S. N. (2000). Structure, function, and control of phosphoinositide-specific phospholipase C. Physiol. Rev. 80, 1291-1335.

Sato, K., Pellegrino, M., Nakagawa, T., Nakagawa, T., Vosshall, L. B., and Touhara, K. (2008). Insect olfactory receptors are heteromeric ligand-gated ion channels. Nature 452

Smart, R., Kiely, A., Beale, M., Vargas, E., Carraher, C., Kralicek, A. V., Christie, D. L., Chen, C., Newcomb, R. D., and Warr, C. G. (2008). Drosophila odorant receptors are novel seven transmembrane domain proteins that can signal independently of heterotrimeric G proteins. Insect Biochem. Mol. Biol. 38, 770-780. 
Stensmyr, M. C., Giordano, E., Balloi, A., Angioy, A. M., and Hansson, B. S. (2003). Novel natural ligands for Drosophila olfactory receptor neurones. J. Exp. Biol. 206, 715-724.

Vosshall, L. B., and Hansson, B. S. (2011). A unified nomenclature system for the insect olfactory co-receptor. Chem. Senses 36, 497-498.

Wicher, D. (2010). Design principles of sensory receptors. Front. Cell. Neurosci. 4:25. doi: 10.3389/fncel.2010.00025

Wicher, D., Schäfer, R., Bauernfeind, R., Stensmyr, M. C., Heller, R., Heinemann, S. H., and Hansson, B. S. (2008). Drosophila odorant receptors are both ligand-gated and cyclic-nucleotide-activated cation channels. Nature 452, 1007-1011.

Yao, C. A., and Carlson, J. R. (2010). Role of G-proteins in odorsensing and CO2-sensing neurons in Drosophila. J. Neurosci. 30, 4562-4572.
Conflict of Interest Statement: The authors declare that the research was conducted in the absence of any commercial or financial relationships that could be construed as a potential conflict of interest.

Received: 24 February 2011; paper pending published: 30 March 2011; accepted: 30 May 2011; published online: 16 June 2011.

Citation: Sargsyan V, Getahun $M N$, Llanos SL, Olsson SB, Hansson BS and Wicher D (2011) Phosphorylation via $P K C$ regulates the function of the Drosophila odorant coreceptor. Front. Cell. Neurosci. 5:5. doi: 10.3389/fncel.2011.00005

Copyright (C) 2011 Sargsyan, Getahun, Llanos, Olsson, Hansson and Wicher. This is an open-access article subject to a non-exclusive license between the authors and Frontiers Media SA, which permits use, distribution and reproduction in other forums, provided the original authors and source are credited and other Frontiers conditions are complied with. 